\title{
Transurethral Enucleation and Resection of the Prostate: Contemporary Role in the Surgical Treatment of Benign Prostatic Hyperplasia
}

\author{
Emad A Alwashmi1 ${ }^{1,2}$ and Abdullah M Alenizi ${ }^{*}$ \\ 1 Division of Urology, Security Forces Hospital, Saudi Arabia \\ ${ }^{2}$ College of medicine, Qassim University, Saudi Arabia
}

Mini Review

Volume 3 Issue 2

Received Date: March 12, 2018

Published Date: March 26, 2018

*Corresponding author: Abdullah M Alenizi, MD, MM Consultant Robotic and MIS Urological Surgeon, Division of Urology, Security Forces Hospital, Riyadh, Saudi Arabia, Postal code 11481, PO Box 3643, Tel: +966118024301; Email: dr.alenizi@hotmail.com

\section{Abstract}

Benign prostatic hyperplasia (BPH) is a very common urological problem affecting all men as they age. Despite the rapid evolution of BPH surgical treatment, transurethral resection of the prostate (TURP) is still considered the gold standard, which has prevailed over the past century. However, due to the safety issues associated with TURP, particularly with prostates larger than $80 \mathrm{ml}$ together with the limited exposure of young urologists to the open prostatectomy, many urologists sought to modify the standard TURP in a way that would assure complete removal of the adenoma with lower risk of complications. Therefore, enucleation was incorporated into the standard TURP in a procedure called transurethral enucleation and resection of the prostate (TUERP), which has been used over the past decade. Besides its ability to provide complete removal of the adenoma, the main advantage of this modification is to help define the capsular plane early during the procedure, which will reduce the risk of capsular perforation and help control bleeders in a timely manner. The technique can be performed with monopolar or bipolar energy. The current evidence proved its safety and efficacy as an alternative to TURP and open prostatectomy in treating medium to large prostate sizes. In this mini review, we discuss the contemporary role of TUERP in the surgical treatment of BPH. We believe that our review will be of great benefit to readers particularly with the rapid evolution of surgical BPH treatment.

Keywords: Benign prostatic enlargement; Surgical treatment; Transurethral resection; Enucleation; Prostatectomy

Abbreviations: AUA: American urological association; AUR: Acute urinary retention; BO0: bladder outlet obstruction; BPH: benign prostatic hyperplasia; EAU: European association of urology; HoLEP: holmium laser enucleation of the prostate; IPSS: International Prostate Symptoms Score; LUTS: lower urinary tract symptoms; PVR: Post-void residual; PSA: prostate specific antigen; TURP: transurethral resection of the prostate; TUERP: 
transurethral enucleation and resection of the prostate; TURS: transurethral resection syndrome; Qmax: maximum flow rate

\section{Introduction}

Benign prostatic hyperplasia (BPH) is a very common urological problem in men as they age. Its histological prevalence in autopsy studies has been found to be $50 \%$ and $80 \%$ in the 6th and 9th decades of life respectively [1]. Management options and decision-making should be guided by severity of lower urinary tract symptoms (LUTS), degree of bother and patient preference [2]. International Prostate Symptoms Score (IPSS) and other scores are frequently used for categorizing treatment options to watchful waiting, life style modification, medical or surgical management [2]. Moreover, according to several guidelines, surgery is indicated in case of Acute urinary retention (AUR) that fails voiding trial, recurrent gross hematuria, recurrent urinary tract infections, bladder stones, bladder diverticulum or renal insufficiency secondary to obstruction [3-5].

Over the past century, transurethral resection of the prostate (TURP) has prevailed as the gold standard surgical treatment for relieving bladder outlet obstruction (BOO) secondary to BPH [3]. However, it has been associated with several perioperative complications including hematuria, blood transfusion, transurethral resection syndrome (TURS) and clot retention particularly with prostates larger than $80 \mathrm{ml}$ [6]. In addition, TURP has been associated with a $3 \%-15 \%$ reoperation rate [7]. Although improvements in training and technology have lead to significant reduction in the rate of these complications, mortality and morbidity are still concerning particularly when operating on high risk patients such as those with cardiac disease and/or anticoagulation therapy [7]. Therefore, the American urological association (AUA) and European association of urology (EAU) guidelines recommend open prostatectomy as the treatment of choice for prostates $>80 \mathrm{ml}$.

Due to the extensive use of medical BPH treatment, the rate of prostate surgery has considerably fallen $[8,9]$. However, when surgery is eventually required, patients are significantly older with more comorbidity [10], which makes them unable to tolerate complications like bleeding and TURS. In addition, due to the widespread use of endoscopic approaches in the past few decades $[11,12]$ the new generation of urologists are graduating with limited exposure on the open approach [13]. As a result, several alternative transurethral techniques have emerged in trials to provide similar functional outcomes with fewer complications as compared to TURP and open prostatectomy. Among those techniques comes the holmium laser enucleation of the prostate (HoLEP), which has shown a comparable functional outcome to the open prostatectomy in treating prostates larger than $80 \mathrm{ml}$ [14]. However, due to reasons related to its steep learning curve and cost $[15,16]$ HoLEP did not gain widespread popularity.

Given the suitability of TURP to all urologists in term of its learning curve, availability and cost effectiveness [17], many urologists sought to incorporate the enucleation technique into the standard TURP. The result would be a modified procedure; namely transurethral enucleation and resection of the prostate (TUERP). The main concept of this modification is to help define the capsular plane early during the procedure particularly at 2-5 and 7-10 o'clock positions of the bladder neck where most bleeders are located [18]. This would allow an under-vision onestep control of those bleeders that will help achieve a bloodless resection of the enucleated adenoma in a timely manner thereby reducing resection time and preventing complications particularly bleeding $[18,19]$. This is difficult to do during the standard TURP where vessels are repeatedly cut before reaching the capsule $[20,21]$.

Being just a modification of the standard TURP, TUERP did not seem to impose any extra cost $[22,23]$. The technique was first described in 1989 by Hiraoka, et al. from Japan [24], which included the use of a detaching blade for the enucleation part that is inserted through the scope and aided by a digital rectal guidance. However, the technique did not gain popularity because of its complexity. In 2010 Liu and colleagues re-described the TUERP using plasma kinetics in which they performed the enucleation by a blunt dissection utilizing the beak of the resectoscope sheath in a retrograde fashion [25]. Due its ease of application that resembles the finger movement during open prostatectomy, many urologists adopted the technique, which lead to a plethora of studies.

\section{Clinical Outcomes}

When assessing the outcome of any new surgical technique, it is essential to compare it with the gold standard with intent to prove a non-inferiority profile. Despite the theoretical idea behind TUERP, some concern still exists regarding the monopolar version of the technique as it relies on hypotonic irrigation fluids that may cause absorption and TURS. Although Hiraoka has 
described a similar technique more than 25 years ago, literature is still scarce when it comes to monopolar TUERP. To the best of our knowledge, the only study in the literature that assessed the difference between monopolar TURP and monopolar TUERP is the one reported by Zuo, et al. in 2014 [26]. In that study, around 600 patients were randomized to receive either TUERP or TURP. Both techniques achieved similar functional outcomes. However, TUERP was superior in terms of resection rate, operative time, irrigation time and blood loss. In addition, no significant drop has been noticed in the postoperative levels of sodium and hemoglobin in the TUERP group. This indicates that the risk of TURS in the monopolar TUERP, even if present, is much lower than that of the TURP counterpart.

When using plasma kinetics, Liu et al concluded that TUERP is a good alternative to TURP and Open simple prostatectomy even for prostate sizes up to $250 \mathrm{ml}$ [25]. However, this conclusion was not based on a direct comparison between these 3 different surgical options. A retrospective study from China looked at the outcome of bipolar TUERP versus bipolar TURP [21]. They reported comparable functional outcomes between the two techniques. However, TUERP was superior in terms of amount of removed tissue and improvement in prostate specific antigen (PSA) and post-void residual (PVR). Although functional outcomes were comparable, the significant difference in the amount of removed tissue and postoperative PSA and PVR may support a more durable symptom relieve in favor of TUERP. Another retrospective study assessed the outcome of bipolar TUERP versus bipolar TURP for prostates larger than $60 \mathrm{ml}$ [27]. In that study TUERP had a better outcome in terms of operative time, irrigation time and hospital stay. In addition, TUERP was also superior in terms of functional outcome including IPSS and maximum flow rate (Qmax), amount of resected tissue and complication rate. However, both studies were retrospective and non-randomized, which may impose a negative impact on their statistical power.

To eliminate the chance of any bias, a prospective study by Luo et al randomized 310 patients to either bipolar TUERP or bipolar TURP [28]. Patients were further categorized according to prostate size to either $<60 \mathrm{ml}$ or $>60 \mathrm{ml}$. functional outcomes were comparable between both approaches however, TURP was associated with longer operative time and more blood loss for prostates $>60 \mathrm{ml}$. To further elaborate on the role of TUERP in treating larger prostate sizes, another prospective randomized trial looked at the outcome of bipolar TUERP versus transvesical prostatectomy for prostates larger than $80 \mathrm{ml}$ [29]. Functional outcomes and complication rate were comparable between the 2 groups. However, TUERP was superior in terms of catheterization time and hospital stay.

Another important aspect in assessing the outcome of TUERP is to compare it with other enucleation techniques. A prospective randomized trial by Gilling group looked at the outcome of plasma kinetic enucleaion of the prostate versus HoLEP [30]. Although functional outcomes were comparable between the two approaches, HoLEP was superior in terms of operative time and catheterization time. However, these results have to be interpreted with caution as all patients in the bipolar enucleation group had morcellation instead of resection, which has been proven to prolong operative time [31].

In summary, due to the relatively recent development of TUERP [25], the current literature lacks long term results. However, short-term data (2 years) proved that TUERP is a safe and feasible alternative to TURP and open prostatectomy in treating medium and large prostate sizes with more tissue removal, shorter operative time, shorter hospital stay and lower complication rate. In addition, its functional outcomes have been shown to be comparable to that of the HoLEP.

\section{Complications}

TUERP shares several TURP complications. The type and rate of those complications depend on several factors including prostate size, comorbidities, duration of surgery, type of electro-cautery and surgeon's experience. These complications include TURS, infection, bleeding requiring blood transfusion, urethral stricture, bladder neck contracture, reoperation and transient incontinence $[29,32,33]$.

Due to the use of hypotonic fluid irrigation, monopolar TUERP is hypothetically expected to carry a similar risk of TURS as its TURP counterpart. However, when prospectively compared with monopolar TURP, TUERP has shown a very low risk of such complication [26]. This observation could be explained by its shorter operative time, early control of bleeders during enucleation and lower risk of capsular perforation. Furthermore, with the advent of plasma kinetics, the risk of TUR-syndrome with TUERP has further declined to negligible values $[27,28,34]$. Moreover, blood loss is another frequent complication that has been shown by several studies to be less frequent with TUERP than with TURP or open prostatectomy $[28,29,34]$. This can be explained by the 
early control of bleeders as they exit the surgical capsule towards the adenoma $[18,19]$.

A major concern during TURP is capsular perforation. Therefore, many urologists avoid deep resection, which may lead to a considerable amount of residual adenoma. This can probably explain the reported 3\%-15\% 5-year reoperation rate after TURP. However, because TUERP provides complete removal of adenoma, its rate of reoperation has been reported to be $0 \%$ at 2 years postoperatively [27].

One of the most commonly reported TUERP complications is transient incontinence, which occurs in $4.7 \%-17 \%$ of cases [28,32-34]. Although stress type has been reported, urge-related incontinence was more common in the majority of cases [28,32]. Nevertheless, almost all patients experience gradual improvement over a period of 3-6 months $[32,33]$. Moreover, looking at the rate of urethral stricture and bladder neck contracture, no difference has been noticed between TUERP and TURP [27].

\section{Conclusion and Future Considerations}

Despite its recent development, TUERP has been proven as a safe alternative to TURP in treating medium to large prostate sizes with more tissue removal, shorter operative time and lower complication rate. It has also shown functional outcomes that are comparable to those of HoLEP and open prostatectomy. Complications are mild and occur less frequently compared to those of the standard TURP and open prostatectomy. In addition, the technique did not seem to impose any extra cost, as it is just a modification of the conventional TURP. It utilizes readily available instruments that all urologists are familiar with, which makes it easy to learn. However, long-term data are still required to validate the current results. Nevertheless, based on the available evidence, we believe that TUERP, particularly bipolar, will soon surpass the conventional TURP as the new gold standard surgical treatment of $\mathrm{BPH}$.

\section{References}

1. Berry SJ, Coffey DS, Walsh PC, Ewing LL (1984) The development of human benign prostatic hyperplasia with age. J Urol 132(3): 474-479.

2. Nickel JC, Mendez-Probst CE, Whelan TF, Paterson RF, Razvi H (2010) 2010 Update: Guidelines for the management of benign prostatic hyperplasia. Can Urol Assoc J 4(5): 310-316.

3. Nimeh T, Magnan B, Almallah YZ (2016) Benign Prostatic Hyperplasia: Review of Modern Minimally Invasive Surgical Treatments. Semin Intervent Radiol 33(3): 244-250.

4. McVary KT, Roehrborn CG, Avins AL, Bruskewitz RC, Donnell RF, et al. (2011) Update on AUA guideline on the management of benign prostatic hyperplasia. J Urol 185(5): 1793-1803.

5. Oelke M, Bachmann A, Descazeaud A, Emberton M, Gravas S, et al. (2013) EAU guidelines on the treatment and follow-up of non-neurogenic male lower urinary tract symptoms including benign prostatic obstruction. Eur Urol 64(1): 118-140.

6. Reich O, Gratzke C, Bachmann A, Seitz M, Schlenker B, et al. (2008) Morbidity, mortality and early outcome of transurethral resection of the prostate: a prospective multicenter evaluation of 10,654 patients. J Urol 180(1): 246-249.

7. Rassweiler J, Teber D, Kuntz R, Hofmann R (2006) Complications of transurethral resection of the prostate (TURP)--incidence, management, and prevention. Eur Urol 50(5): 969-979.

8. Izard J, Nickel JC (2011) Impact of medical therapy on transurethral resection of the prostate: two decades of change. BJU Int 108(1): 89-93.

9. Malaeb BS, Yu X, McBean AM, Elliott SP (2012) National trends in surgical therapy for benign prostatic hyperplasia in the United States (20002008). Urology 79(5): 1111-1116.

10. Descazeaud A, Robert G, Azzousi AR, Ballereau C, Lukacs B, et al. (2009) Laser treatment of benign prostatic hyperplasia in patients on oral anticoagulant therapy: a review. BJU Int 103(9): 1162-1165.

11. Hueber PA, Zorn KC (2013) Canadian trend in surgical management of benign prostatic hyperplasia and laser therapy from 2007-2008 to 2011-2012. Can Urol Assoc J 7(9-10): E582-586.

12. Lowrance WT, Southwick A, Maschino AC, Sandhu JS (2013) Contemporary practice patterns of endoscopic surgical management for benign prostatic 
hyperplasia among urologists in the United States. J Urol 189(5): 1811-1816.

13. Parsa CJ, Organ CH, Jr, Barkan H (2000) Changing patterns of resident operative experience from 1990 to 1997. Arch Surg 135(5): 570-573.

14. Chen H, Tang P, Ou R, Deng X, Xie K (2012) Holmium laser enucleation versus open prostatectomy for large volume benign prostatic hyperplasia: a meta-analysis of the therapeutic effect and safety. Nan Fang Yi Ke Da Xue Xue Bao 32(6): 882-885.

15. Elzayat EA, Elhilali MM (2007) Holmium laser enucleation of the prostate (HoLEP): long-term results, reoperation rate, and possible impact of the learning curve. Eur Urol 52(5): 1465-1471.

16. Robert G, Cornu JN, Fourmarier M, Saussine C, Descazeaud A, et al. (2016) Multicentre prospective evaluation of the learning curve of holmium laser enucleation of the prostate (HoLEP). BJU Int 117(3): 495-499.

17. Lourenco T, Armstrong N, N'Dow J, Nabi G, Deverill M, et al. (2008) Systematic review and economic modelling of effectiveness and cost utility of surgical treatments for men with benign prostatic enlargement. Health Technol Assess 12(35): 169-515.

18. Choo MS, Lee HE, Bae J, Cho SY, Oh SJ (2014) Transurethral surgical anatomy of the arterial bleeder in the enucleated capsular plane of enlarged prostates during holmium laser enucleation of the prostate. Int Neurourol J 18(3): 138-144.

19. Zhao Z, Zeng G, Zhong W, Mai Z, Zeng S, et al. (2010) A prospective, randomised trial comparing plasmakinetic enucleation to standard transurethral resection of the prostate for symptomatic benign prostatic hyperplasia: three-year follow-up results. Eur Urol 58(5): 752-758.

20. Hiraoka Y, Akimoto M (1987) Anatomy of the prostate from fetus to adult--origin of benign prostatic hyperplasia. Urol Res 15(3): 177-180.

21. Zhang KY, Xing JC, Chen BS, Liu CX, Lau HW, et al. (2011) Bipolar plasmakinetic transurethral resection of the prostate vs. transurethral enucleation and resection of the prostate: pre- and postoperative comparisons of parameters used in assessing benign prostatic enlargement. Singapore Med 52(10): 747687514.
22. Xu A, Zou Y, Li B, Zheng S, Li H, et al. (2013) A randomized trial comparing diode laser enucleation of the prostate with plasmakinetic enucleation and resection of the prostate for the treatment of benign prostatic hyperplasia. J Endourol 27(10): 1254-1260.

23. Chen YXH, Xu H, Gu M, Zhou J, Cai Z, et al. (2016) Comparison of plasmakinetic enucleation of the prostate with holmium laser enucleation of the prostate in the treatment of benign prostate hyperplasia. Int J Clin Exp Med 9: 7328-7333.

24. Hiraoka Y, Akimoto M (1989) Transurethral enucleation of benign prostatic hyperplasia. J Urol 142(5): 1247-1250.

25. Liu C, Zheng S, Li H, Xu K (2010) Transurethral enucleation and resection of prostate in patients with benign prostatic hyperplasia by plasma kinetics. J Urol 184(6): 2440-2445.

26. Zuo W, Wang ZZ, Xue J (2014) Transurethral enucleative resection of the prostate versus transurethral resection of the prostate for benign prostate hyperplasia. Zhonghua Nan Ke Xue 20(9): 812-815.

27. Wei Y, Xu N, Chen SH, Li XD, Zheng QS, et al. (2016) Bipolar transurethral enucleation and resection of the prostate versus bipolar resection of the prostate for prostates larger than 60gr: A retrospective study at a single academic tertiary care center. Int Braz J Urol 42(4): 747-756.

28. Luo YH, Shen JH, Guan RY, Li H, Wang J (2014) Plasmakinetic enucleation of the prostate vs plasmakinetic resection of the prostate for benign prostatic hyperplasia: comparison of outcomes according to prostate size in 310 patients. Urology 84(4): 904-910.

29. Ou R, Deng X, Yang W, Wei X, Chen H, et al. (2013) Transurethral enucleation and resection of the prostate vs transvesical prostatectomy for prostate volumes $>80 \mathrm{~mL}$ : a prospective randomized study. BJU Int 112(2): 239-245.

30. Neill MG, Gilling PJ, Kennett KM, Frampton CM, Westenberg AM, et al. (2006) Randomized trial comparing holmium laser enucleation of prostate with plasmakinetic enucleation of prostate for treatment of benign prostatic hyperplasia. Urology 68(5): 1020-1024. 
31. Chen YB, Chen Q, Wang Z, Yu-Bing Peng, Li-Ming Ma, et al. (2013) A prospective, randomized clinical trial comparing plasmakinetic resection of the prostate with holmium laser enucleation of the prostate based on a 2-year followup. J Urol 189(1): 217-222.

32. Palaniappan S, Kuo TL, Cheng CW, Foo KT (2016) Early outcome of transurethral enucleation and resection of the prostate versus transurethral resection of the prostate. Singapore Med J 57(12): 676-680.
33. Xu P, Xu A, Chen B, Zheng S, Xu Y, et al. (2018) Bipolar transurethral enucleation and resection of the prostate: Whether it is ready to supersede TURP? Asian J Urol 5(1): 48-54.

34. Rao JM, Yang JR, Ren YX, He J, Ding P, et al. (2013) Plasmakinetic enucleation of the prostate versus transvesical open prostatectomy for benign prostatic hyperplasia $>80 \mathrm{~mL}$ : 12 -month follow-up results of a randomized clinical trial. Urology 82(1): 176-181. 